Article

\title{
Entropy Transport Equation in Large Eddy Simulation for Exergy Analysis of Turbulent Combustion Systems
}

\author{
Mehdi Safari, M. Reza H. Sheikhi *, Mohammad Janbozorgi and Hameed Metghalchi \\ Department of Mechanical and Industrial Engineering, Northeastern University, 360 Huntington \\ Avenue, Boston, MA 02115, USA \\ * Author to whom correspondence should be addressed; E-Mail: sheikhi@ neu.edu, \\ Tel.: +1-617-373-3427, Fax: +1-617-373-2921.
}

Received: 11 January 2010, in revised form: 20 February 2010 / Accepted: 5 March 2010 /

Published: 8 March 2010

\begin{abstract}
The transport equation of entropy is introduced in large eddy simulation to perform exergy analysis of turbulent combustion systems. The sources of exergy destruction can be evaluated by analyzing entropy generation terms, which appear in unclosed forms in this equation. The closure is based on the filtered density function (FDF) methodology. The primary advantage of FDF is that chemical reaction and its entropy generation effects appear in closed forms. This methodology involves a stochastic model, which is being developed to account for the subgrid scale transport of entropy.
\end{abstract}

Keywords: entropy generation; exergy analysis; large eddy simulation, filtered density function, probability density function, turbulent combustion

\section{Introduction}

Combustion of fuels is still the world's main source of energy and this is projected to continue in the foreseeable future. Considering the limited fuel reserve and the environmental effects of combustion, it is thus of prime importance to enhance the thermal and combustion efficiencies of combustion systems. From the second law of thermodynamics, combustion efficiency is the "quality" at which the thermal energy is available in the hot products of combustion [1,2]. In conventional engineering, the main 
concern is energy conversion without considering the exergy destruction. Analysis of exergy loss and entropy generation, which focuses on the optimal potential work done while bringing a system into equilibrium with an environment is an effective tool to investigate combustion efficiency and irreversibilities in combustion systems [2,3].

The exergy analysis has been the subject of widespread research [1-22]. The vast majority of contributions so far has been on exergy analysis of combustion systems with laminar flow regimes [3,5, $6,11,12,19,20]$. Despite the prevalence of turbulence in practical combustion devices, the application of exergy analysis to turbulent reacting flows has been very limited [8,10,16,18]. These efforts mainly involve solution of Reynolds Averaged Navier-Stokes (RANS) equations, extended to include the transport of entropy $[8,10,18]$. Large eddy simulation (LES) is widely known to be the optimal means of capturing the detailed, unsteady physics of turbulent reacting flows [23-29]. It can thus be utilized as an effective tool to preform exergy analysis of turbulent combustion systems. The primary challenge in LES is the accurate modeling of the subgrid scale (SGS) quantities [25]. The Filtered Density Function (FDF) methodology has proven particularly effective for this purpose [23,30]. The FDF is essentially the counterpart of the Probability Density Function (PDF) method in RANS [23] and has the unique capability to account for chemical reaction and its entropy generation effects in an exact manner. The predictive capabilities of FDF is demonstrated by applying it to several flame geometries [31-33]. The most comprehensive form of FDF to date is the joint frequency-velocity-scalar FDF [34], which accounts for the joint SGS statistics of velocity, thermo-chemical compositions and turbulent frequency. [35] provides a recent review of the state of progress in LES via FDF. In the present study, the sources of exergy destruction are accounted for by analyzing the entropy generation terms in the entropy transport equation. This equation is considered in LES of turbulent reactive flows. The unclosed terms in this equation are all accounted for by the FDF, which is particularly advantageous in providing closed forms for chemical reaction and its entropy generation effects. An exact transport equation is developed here for the FDF, which is represented by a stochastic model. In Section 2, the entropy transport equation and the rate of exergy destruction are obtained from thermodynamic principles. Section 3 involves LES implementation of entropy transport equation and calculation of exergy loss in LES. In Section 4, an FDF methodology to account for the unclosed SGS terms in entropy transport equation is developed.

\section{Theoretical Foundation}

The thermodynamic efficiency of a device or process can be assessed through the measure of the irreversibilities generated, which has widely been evaluated using the second law analysis. Practical energy conversion processes are always accompanied by an irreversible increase in entropy, which leads to a decrease in exergy (available energy). Thus, even though energy is conserved, exergy decreases because energy is converted into a different form, from which less work can be obtained. Reduced entropy generation results in more efficient use of available energy in combustion systems. Therefore, to improve the performance of these systems, exergy analysis is essential to explain the sources of exergy loss. The exergy balance equation reads as:

$$
\frac{d E_{x}}{d t}=\dot{E}_{x_{t}}-\dot{E}_{x_{D}}
$$


where $E_{x}$ is the exergy and the term on the left hand side specifies the rate of change of exergy within the system. $\dot{E}_{x_{t}}$ is the rate of exergy transfer due to heat, work and flow interactions and $\dot{E}_{x_{D}}$ denotes the rate of exergy destruction due to irreversibility and can be expressed as:

$$
\dot{E}_{x_{D}}=T_{0} \dot{S}_{g}
$$

where $T_{0}$ is the ambient (dead state) temperature and $\dot{S}_{g}$ is the rate of entropy generation due to irreversibilities which can be obtained from the transport of entropy, as described below.

In exothermic, chemically reacting, variable density flows, the primary transport variables are the fluid density $\rho(\mathbf{x}, t)$, the velocity vector $u_{i}(\mathbf{x}, t), i=1,2,3$ along the $x_{i}$ direction, the specific enthalpy $h(\mathbf{x}, t)$, the pressure $p(\mathbf{x}, t)$, the mass fractions of $N_{s}$ species, $Y_{\alpha}(\mathbf{x}, t)\left(\alpha=1,2, \ldots, N_{s}\right)$ and the entropy $s(\mathbf{x}, t)$, where $\mathbf{x}=x_{i}(i=1,2,3)$ and $t$ denote space and time, respectively. These variables satisfy the conservation equations of mass, momentum, energy and species mass fractions [36] as well as the transport of entropy, which follows the thermodynamic relation [37]

$$
T \rho \frac{D s}{D t}=\rho \frac{D e}{D t}+p \rho \frac{D v}{D t}-\sum_{\alpha=1}^{N_{s}} \mu_{\alpha} \rho \frac{D Y_{\alpha}}{D t}
$$

where $T, e$ and $v$ denote the temperature, internal energy and the specific volume, respectively. $D / D t=\partial / \partial t+u_{i} \partial / \partial x_{i}$ denotes the material derivative. $\mu_{\alpha}$ denotes the chemical potential (per unit mass of species $\alpha$ ),

$$
\mu_{\alpha}=h_{\alpha}-T s_{\alpha}^{o}
$$

in which,

$$
s_{\alpha}^{o}=\frac{1}{M_{\alpha}}\left(\frac{\partial S}{\partial n_{\alpha}}\right)_{T, p, n_{\beta} \quad(\beta \neq \alpha)}
$$

is the partial entropy, $n_{\alpha}$ and $M_{\alpha}$ are the number of moles and molecular mass of species $\alpha$, respectively. $S$ denotes the (extensive) entropy,

$$
S=m s=m \sum_{\alpha=1}^{N_{s}} Y_{\alpha} s_{\alpha}^{o},
$$

where $m$ is the total mass. For an ideal gas, the partial entropy can be expressed in terms of entropy of pure substance as:

$$
s_{\alpha}^{o}=s_{\alpha}-R_{\alpha} \ln X_{\alpha}
$$

In this equation, $s_{\alpha}$ denotes the entropy of pure species $\alpha, X_{\alpha}$ denotes the mole fraction of species $\alpha$ and $R_{\alpha}$ is the gas constant for species $\alpha\left(R_{\alpha}=R_{u} / M_{\alpha}\right.$ and $R_{u}$ is the universal gas constant). In Equation (4), $h_{\alpha}$ is the enthalpy of species $\alpha$,

$$
h_{\alpha}=h_{\alpha}^{0}+\int_{T_{r}}^{T} c_{p_{\alpha}}\left(T^{\prime}\right) d T^{\prime}
$$


in which $h_{\alpha}^{0}$ denotes the enthalpy of species $\alpha$ at reference temperature $T_{r}$ and $c_{p_{\alpha}}$ represents specific heat at constant pressure for species $\alpha$. By substituting for $D e / D t$ and $D Y_{\alpha} / D t$ from conservation equations of energy and chemical species mass fraction [24], we obtain a transport equation for entropy,

$$
\frac{\partial \rho s}{\partial t}+\frac{\partial \rho u_{i} s}{\partial x_{i}}=\frac{1}{T}\left[\tau_{i j} \frac{\partial u_{i}}{\partial x_{j}}-\frac{\partial \dot{q}_{i}}{\partial x_{i}}+\sum_{\alpha=1}^{N_{s}} \mu_{\alpha} \frac{\partial J_{i}^{\alpha}}{\partial x_{i}}-\sum_{\alpha=1}^{N_{s}} \rho \mu_{\alpha} S_{\omega_{\alpha}}\right]
$$

In this equation, $\tau_{i j}$ and $J_{i}^{\alpha}$ denote the viscous stress tensor and the scalar fluxes, respectively. Assuming a Newtonian fluid and Fick's law of diffusion, these are represented as

$$
\begin{gathered}
\tau_{i j}=\mu\left(\frac{\partial u_{i}}{\partial x_{j}}+\frac{\partial u_{j}}{\partial x_{i}}-\frac{2}{3} \frac{\partial u_{k}}{\partial x_{k}} \delta_{i j}\right) \\
J_{i}^{\alpha}=-\gamma_{\alpha} \frac{\partial Y_{\alpha}}{\partial x_{i}}
\end{gathered}
$$

where $\mu$ is the molecular viscosity and $\gamma_{\alpha}$ denotes the mass molecular diffusivity of species $\alpha$. In Equation (9), $S_{\omega_{\alpha}}$ is a source term due to chemical reaction, defined as

$$
S_{\omega_{\alpha}}=\frac{1}{\rho} M_{\alpha} \dot{\omega}_{\alpha}
$$

where $\dot{\omega}_{\alpha}$ is the molar rate of production/destruction of species $\alpha$ due to chemical reaction. Also in Equation (9), $\dot{q}_{i}$ is the energy flux term, expressed as

$$
\begin{aligned}
\dot{q}_{i} & =-\lambda \frac{\partial T}{\partial x_{i}}+\sum_{\alpha=1}^{N_{s}} h_{\alpha} J_{i}^{\alpha} \\
& =-\lambda \frac{\partial T}{\partial x_{i}}-\sum_{\alpha=1}^{N_{s}} \gamma_{\alpha} h_{\alpha} \frac{\partial Y_{\alpha}}{\partial x_{i}}
\end{aligned}
$$

where the rate of heat transfer by radiation is neglected. In this equation $\lambda$ denotes the thermal conductivity. The entropy transport equation, Equation (9), can be written in a form consistent with that in [37],

$$
\begin{aligned}
\frac{\partial \rho s}{\partial t}+\frac{\partial \rho u_{i} s}{\partial x_{i}} & =\frac{1}{T}[\tau_{i j} \frac{\partial u_{i}}{\partial x_{j}}-\frac{1}{T}\left(\dot{q}_{i}-\sum_{\alpha=1}^{N_{s}} h_{\alpha} J_{i}^{\alpha}\right) \frac{\partial T}{\partial x_{i}}-\sum_{\alpha=1}^{N_{s}} \rho \mu_{\alpha} S_{\omega_{\alpha}} \underbrace{\sum_{\alpha=1}^{N_{s}} J_{i}^{\alpha}\left(\frac{\partial \mu_{\alpha}}{\partial x_{i}}+s_{\alpha}^{o} \frac{\partial T}{\partial x_{i}}\right)}_{I V}] \\
& \underbrace{-\frac{\partial}{\partial x_{i}}\left[\frac{1}{T}\left(\dot{q}_{i}-\sum_{\alpha=1}^{N_{s}} h_{\alpha} J_{i}^{\alpha}\right)+\sum_{\alpha=1}^{N_{s}} s_{\alpha}^{o} J_{i}^{\alpha}\right]}_{V}
\end{aligned}
$$


Utilizing Equation (8), the term $I V$ on the right hand side (RHS) of this equation can be expressed as:

$$
-\sum_{\alpha=1}^{N_{s}} \frac{J_{i}^{\alpha}}{T}\left(\frac{\partial \mu_{\alpha}}{\partial x_{i}}+s_{\alpha}^{o} \frac{\partial T}{\partial x_{i}}\right)=-\sum_{\alpha=1}^{N_{s}} \frac{J_{i}^{\alpha}}{T}\left(c_{p_{\alpha}} \frac{\partial T}{\partial x_{i}}-T \frac{\partial s_{\alpha}^{o}}{\partial x_{i}}\right)
$$

For an ideal gas, taking derivative of Equation (7) results in,

$$
\frac{\partial s_{\alpha}^{o}}{\partial x_{i}}=\frac{1}{T} c_{p_{\alpha}} \frac{\partial T}{\partial x_{i}}-\frac{R_{\alpha}}{Y_{\alpha}} \frac{\partial Y_{\alpha}}{\partial x_{i}}+\frac{R_{\alpha}}{n} \frac{\partial n}{\partial x_{i}}-\overbrace{\frac{R_{\alpha}}{p} \frac{\partial p}{\partial x_{i}}}^{\approx 0}
$$

where the last term can be neglected in low Mach number combustion processes $[10,23,38]$. By employing Equation (16), the term $I V$ in Equation (14) can thus be written as:

$$
-\sum_{\alpha=1}^{N_{s}} \frac{J_{i}^{\alpha}}{T}\left(\frac{\partial \mu_{\alpha}}{\partial x_{i}}+s_{\alpha}^{o} \frac{\partial T}{\partial x_{i}}\right)=-\sum_{\alpha=1}^{N_{s}} R_{\alpha} J_{i}^{\alpha}\left(\frac{1}{Y_{\alpha}} \frac{\partial Y_{\alpha}}{\partial x_{i}}-\frac{1}{n} \frac{\partial n}{\partial x_{i}}\right)
$$

In Equation (14), the term $V$ can be further simplified by using Equations $(11,13)$ as:

$$
-\frac{\partial}{\partial x_{i}}\left[\frac{1}{T}\left(\dot{q}_{i}-\sum_{\alpha=1}^{N_{s}} h_{\alpha} J_{i}^{\alpha}\right)+\sum_{\alpha=1}^{N_{s}} s_{\alpha}^{o} J_{i}^{\alpha}\right]=\frac{\partial}{\partial x_{i}}\left[\frac{\lambda}{T} \frac{\partial T}{\partial x_{i}}+\sum_{\alpha=1}^{N_{s}} \gamma_{\alpha} s_{\alpha}^{o} \frac{\partial Y_{\alpha}}{\partial x_{i}}\right]
$$

This equation can further be simplified by employing Equation (16) and assuming equal mass diffusivity for all species $\left(\gamma_{\alpha}=\gamma, \alpha=1, \ldots, N_{s}\right)$ and unity Lewis number $\left(L e=\lambda /\left(\gamma c_{p}\right)=1\right.$, where $c_{p}=\sum_{\alpha=1}^{N_{s}} c_{p_{\alpha}} Y_{\alpha}$ ). Therefore, the term $V$ in Equation (14) reduces to diffusion of entropy:

$$
-\frac{\partial}{\partial x_{i}}\left[\frac{1}{T}\left(\dot{q}_{i}-\sum_{\alpha=1}^{N_{s}} h_{\alpha} J_{i}^{\alpha}\right)+\sum_{\alpha=1}^{N_{s}} s_{\alpha}^{o} J_{i}^{\alpha}\right]=\frac{\partial}{\partial x_{i}}\left(\gamma \frac{\partial s}{\partial x_{i}}\right)
$$

By substituting Equations $(17,19)$ into Equation (14), another useful form of entropy transport equation can be obtained,

$$
\begin{aligned}
\frac{\partial \rho s}{\partial t}+\frac{\partial \rho u_{i} s}{\partial x_{i}} & =\frac{\partial}{\partial x_{i}}\left(\gamma \frac{\partial s}{\partial x_{i}}\right)+\frac{1}{T} \tau_{i j} \frac{\partial u_{i}}{\partial x_{j}}-\frac{\rho}{T} \sum_{\alpha=1}^{N_{s}} \mu_{\alpha} S_{\omega_{\alpha}}+\frac{\lambda}{T^{2}} \frac{\partial T}{\partial x_{i}} \frac{\partial T}{\partial x_{i}} \\
& +\sum_{\alpha=1}^{N_{s}} \gamma R_{\alpha} \frac{1}{Y_{\alpha}} \frac{\partial Y_{\alpha}}{\partial x_{i}} \frac{\partial Y_{\alpha}}{\partial x_{i}}-\sum_{\alpha=1}^{N_{s}} \gamma R_{\alpha} \frac{1}{n} \frac{\partial Y_{\alpha}}{\partial x_{i}} \frac{\partial n}{\partial x_{i}}
\end{aligned}
$$

In this equation, the last five terms on the RHS constitute the volumetric rate of generation of entropy $S^{\prime \prime \prime \prime} g$. Therefore,

$$
\dot{S}^{\prime \prime \prime \prime}{ }_{g}=\frac{1}{T} \tau_{i j} \frac{\partial u_{i}}{\partial x_{j}}-\frac{\rho}{T} \sum_{\alpha=1}^{N_{s}} \mu_{\alpha} S_{\omega_{\alpha}}+\frac{\lambda}{T^{2}} \frac{\partial T}{\partial x_{i}} \frac{\partial T}{\partial x_{i}}+\sum_{\alpha=1}^{N_{s}} \gamma R_{\alpha} \frac{1}{Y_{\alpha}} \frac{\partial Y_{\alpha}}{\partial x_{i}} \frac{\partial Y_{\alpha}}{\partial x_{i}}-\sum_{\alpha=1}^{N_{s}} \gamma R_{\alpha} \frac{1}{n} \frac{\partial Y_{\alpha}}{\partial x_{i}} \frac{\partial n}{\partial x_{i}}
$$

As this equation demonstrates, the irreversible processes involved in entropy generation are: viscous dissipation, chemical reaction, heat conduction, mass transfer due to change in mass fraction of 
individual species and mass transfer due to change in total number of moles. Similar transport equations are considered in $[10,18]$ to perform exergy analysis of turbulent diffusion flames via RANS. Due to capacity of LES to predict instantaneous flow characteristics and resolve turbulent flow structures, in this work the entropy transport is implemented in LES to facilitate more accurate prediction of exergy losses in turbulent reactive flows.

\section{Entropy Transport in Large Eddy Simulation}

We consider LES implementation of entropy transport equation along with conservation equations of mass, momentum, energy and species mass fractions. LES involves the spatial filtering operation $[23,39,40]$

$$
\langle Q(\mathbf{x}, t)\rangle=\int_{-\infty}^{+\infty} Q\left(\mathbf{x}^{\prime}, t\right) \mathcal{G}\left(\mathbf{x}^{\prime}, \mathbf{x}\right) d \mathbf{x}^{\prime}
$$

where $\mathcal{G}$ denotes the filter function of width $\Delta_{\mathcal{G}}$, and $\langle Q(\mathbf{x}, t)\rangle$ represents the filtered value of the transport variable $Q(\mathbf{x}, t)$. In reacting flows, it is convenient to consider the Favre filtered quantity, $\langle Q(\mathbf{x}, t)\rangle_{L}=\langle\rho Q\rangle /\langle\rho\rangle$. We consider spatially invariant and localized filter functions, $\mathcal{G}\left(\mathbf{x}^{\prime}, \mathbf{x}\right) \equiv \mathcal{G}\left(\mathbf{x}^{\prime}-\mathbf{x}\right)$ with the properties [41] $\mathcal{G}(\mathbf{x})=\mathcal{G}(-\mathbf{x})$, and $\int_{-\infty}^{\infty} \mathcal{G}(\mathbf{x}) d \mathbf{x}=1$. We consider "positive" filter functions [42] for which all the moments $\int_{-\infty}^{\infty} x^{m} \mathcal{G}(x) d x$ exist for $m \geq 0$. The filtered variables satisfy the filtered form of conservation equations of mass, momentum, energy and species mass fractions [43] as well as entropy transport equation. This equation is obtained by filtering Equation (20) according to Equation (22) as

$$
\frac{\partial\langle\rho\rangle\langle s\rangle_{L}}{\partial t}+\frac{\partial\langle\rho\rangle\left\langle u_{i}\right\rangle_{L}\langle s\rangle_{L}}{\partial x_{i}}=\frac{\partial}{\partial x_{i}}\left(\gamma \frac{\partial\langle s\rangle_{L}}{\partial x_{i}}\right)-\frac{\partial\langle\rho\rangle \tau\left(u_{i}, s\right)}{\partial x_{i}}+\left\langle\dot{S}^{\prime \prime \prime \prime}{ }_{g}\right\rangle
$$

where

$$
\tau(a, b)=\langle a b\rangle_{L}-\langle a\rangle_{L}\langle b\rangle_{L}
$$

denotes the second-order SGS correlations. The last two terms on the RHS of Equation (23), representing the SGS entropy flux and the filtered volumetric rate of entropy generation, are unclosed. The filtered entropy generation term can be expressed as the filtered form of individual irreversible processes, according to Equation (21):

$$
\begin{aligned}
\left\langle\dot{S}_{g}^{\prime \prime \prime}{ }_{g}\right\rangle & =\left\langle\frac{1}{T} \tau_{i j} \frac{\partial u_{i}}{\partial x_{j}}\right\rangle-\left\langle\frac{\rho}{T} \sum_{\alpha=1}^{N_{s}} \mu_{\alpha} S_{\omega_{\alpha}}\right\rangle+\left\langle\frac{\lambda}{T^{2}} \frac{\partial T}{\partial x_{i}} \frac{\partial T}{\partial x_{i}}\right\rangle+\left\langle\sum_{\alpha=1}^{N_{s}} \gamma R_{\alpha} \frac{1}{Y_{\alpha}} \frac{\partial Y_{\alpha}}{\partial x_{i}} \frac{\partial Y_{\alpha}}{\partial x_{i}}\right\rangle \\
& -\left\langle\sum_{\alpha=1}^{N_{s}} \gamma R_{\alpha} \frac{1}{n} \frac{\partial Y_{\alpha}}{\partial x_{i}} \frac{\partial n}{\partial x_{i}}\right\rangle
\end{aligned}
$$

These terms are all accounted for by the methodology described in the next section. By calculating the filtered entropy generation term, the filtered rate of exergy destruction can consequently be obtained according to Equation (2). 


\section{The Filtered Density Function Methodology}

In this section, the filtered density function (FDF) methodology is developed to account for the unclosed terms in the entropy transport equation. This methodology contains the complete statistical information about the joint SGS variation of velocity, scalar, frequency and entropy fields [30,34]. This form of FDF, denoted by $\mathcal{F}(\boldsymbol{v}, \boldsymbol{\psi}, \theta, r, \mathbf{x} ; t)$, is defined by

$$
\mathcal{F}(\boldsymbol{v}, \boldsymbol{\psi}, \theta, r, \mathbf{x} ; t)=\int_{-\infty}^{+\infty} \rho\left(\mathbf{x}^{\prime}, t\right) \xi\left[\boldsymbol{v}, \boldsymbol{\psi}, \theta, r ; \boldsymbol{u}\left(\mathbf{x}^{\prime}, t\right), \boldsymbol{\phi}\left(\mathbf{x}^{\prime}, t\right), \omega\left(\mathbf{x}^{\prime}, t\right), s\left(\mathbf{x}^{\prime}, t\right)\right] \mathcal{G}\left(\mathbf{x}^{\prime}-\mathbf{x}\right) d \mathbf{x}^{\prime}
$$

where

$$
\begin{aligned}
& \xi[\boldsymbol{v}, \boldsymbol{\psi}, \theta, r ; \boldsymbol{u}(\mathbf{x}, t), \boldsymbol{\phi}(\mathbf{x}, t), \omega(\mathbf{x}, t), s(\mathbf{x}, t)]= \\
& \delta(r-s(\mathbf{x}, t)) \delta(\theta-\omega(\mathbf{x}, t)) \times \prod_{i=1}^{3} \delta\left(v_{i}-u_{i}(\mathbf{x}, t)\right) \prod_{\alpha=1}^{N_{s}+1} \delta\left(\psi_{\alpha}-\phi_{\alpha}(\mathbf{x}, t)\right)
\end{aligned}
$$

is the "fine-grained" density $[23,44,45]$ and $\delta$ denotes the Dirac delta function. The sample space variables $\boldsymbol{v}, \boldsymbol{\psi}, \theta$ and $r$ correspond to the velocity $\boldsymbol{u}(\mathbf{x}, t)$, the scalar array $\boldsymbol{\phi}(\mathbf{x}, t)=\left[Y_{1}, \ldots, Y_{N_{s}}, h\right]$, the SGS frequency $\omega(\mathbf{x}, t)$ and the entropy $s(\mathbf{x}, t)$, respectively. Following the procedure detailed in $[34,43,46]$, the FDF satisfies an exact transport equation,

$$
\begin{aligned}
& \frac{\partial \mathcal{F}}{\partial t}+\frac{\partial\left(v_{i} \mathcal{F}\right)}{\partial x_{i}}=-\sum_{\alpha=1}^{N_{s}} \frac{\partial}{\partial \psi_{\alpha}}\left[S_{\omega_{\alpha}}(\boldsymbol{\psi}) \mathcal{F}\right]+\frac{\partial}{\partial r}\left[\frac{1}{T} \sum_{\alpha=1}^{N_{s}} \mu_{\alpha}(\boldsymbol{\psi}) S_{\omega_{\alpha}}(\boldsymbol{\psi}) \mathcal{F}\right] \\
+ & \frac{\partial}{\partial v_{i}}\left[\left\langle\frac{1}{\rho} \frac{\partial p}{\partial x_{i}} \mid \boldsymbol{v}, \boldsymbol{\psi}, \theta, r\right\rangle \mathcal{F}\right]-\frac{\partial}{\partial v_{i}}\left[\left\langle\frac{1}{\rho} \frac{\partial \tau_{i j}}{\partial x_{j}} \mid \boldsymbol{v}, \boldsymbol{\psi}, \theta, r\right\rangle \mathcal{F}\right] \\
+ & \sum_{\alpha=1}^{N_{s}} \frac{\partial}{\partial \psi_{\alpha}}\left[\left\langle\frac{1}{\rho} \frac{\partial J_{i}^{\alpha}}{\partial x_{i}} \mid \boldsymbol{v}, \boldsymbol{\psi}, \theta, r\right\rangle \mathcal{F}\right]-\frac{\partial}{\partial \theta}\left[\left\langle\frac{D \omega}{D t} \mid \boldsymbol{v}, \boldsymbol{\psi}, \theta, r\right\rangle \mathcal{F}\right] \\
- & \frac{\partial}{\partial r}\left[\left\langle\frac{1}{\rho} \frac{\partial}{\partial x_{i}}\left(\gamma \frac{\partial s}{\partial x_{i}}\right) \mid \boldsymbol{v}, \boldsymbol{\psi}, \theta, r\right\rangle \mathcal{F}\right] \\
- & \frac{\partial}{\partial r}\left[\left\langle\frac{1}{\rho T} \tau_{i j} \frac{\partial u_{i}}{\partial x_{j}} \mid \boldsymbol{v}, \boldsymbol{\psi}, \theta, r\right\rangle \mathcal{F}\right]-\frac{\partial}{\partial r}\left[\left\langle\frac{\lambda}{\rho T^{2}} \frac{\partial T}{\partial x_{i}} \frac{\partial T}{\partial x_{i}} \mid \boldsymbol{v}, \boldsymbol{\psi}, \theta, r\right\rangle \mathcal{F}\right] \\
- & \frac{\partial}{\partial r}\left[\left\langle\frac{1}{\rho} \sum_{\alpha=1}^{N_{s}} \gamma R_{\alpha} \frac{1}{Y_{\alpha}} \frac{\partial Y_{\alpha}}{\partial x_{i}} \frac{\partial Y_{\alpha}}{\partial x_{i}} \mid \boldsymbol{v}, \boldsymbol{\psi}, \theta, r\right\rangle \mathcal{F}\right]+\frac{\partial}{\partial r}\left[\left\langle\frac{1}{\rho} \sum_{\alpha=1}^{N_{s}} \gamma R_{\alpha} \frac{1}{n} \frac{\partial Y_{\alpha}}{\partial x_{i}} \frac{\partial n}{\partial x_{i}} \mid \boldsymbol{v}, \boldsymbol{\psi}, \theta, r\right\rangle \mathcal{F}\right]
\end{aligned}
$$

where $\langle\mid\rangle$ denotes the "conditional" filtered values. As this equation shows, the effects of SGS convection (the second term on the left hand side), combustion (the first term on the RHS) and the entropy generation due to combustion (the second term on the RHS) are all in closed forms. However, 
all of the terms involving conditional filtered values require closures. The filtered value of any function $\hat{Q}$ of the velocity, scalar, frequency and/or entropy is calculated by

$$
\langle\rho(\mathbf{x}, t)\rangle\langle Q(\mathbf{x}, t)\rangle_{L}=\int_{-\infty}^{+\infty} \hat{Q}(\boldsymbol{v}, \boldsymbol{\psi}, \theta, r) \mathcal{F}(\boldsymbol{v}, \boldsymbol{\psi}, \theta, r, \mathbf{x} ; t) d \boldsymbol{v} d \boldsymbol{\psi} d \theta d r
$$

The unclosed terms in Equation (28) are modeled by a stochastic model which is in the form of coupled stochastic differential equations (SDE). In $[34,43,46]$ the SDEs corresponding to position, velocity, scalar and SGS frequency are constructed. To account for the unclosed terms in the entropy transport equation in this work, the system of SDEs is supplemented with a SDE for entropy, which is currently under development. This SDE follows the thermodynamic laws and has the general form of a diffusion process [47],

$$
d s^{+}(t)=D\left(\mathbf{X}^{+}, \mathbf{U}^{+}, \boldsymbol{\phi}^{+}, s^{+}, \omega^{+} ; t\right) d t+B_{j}\left(\mathbf{X}^{+}, \mathbf{U}^{+}, \boldsymbol{\phi}^{+}, s^{+}, \omega^{+} ; t\right) d W_{j}(t)
$$

where $\mathbf{X}^{+}, \boldsymbol{U}^{+}, \phi^{+}, \omega^{+}$and $s^{+}$are probabilistic representations of position, velocity vector, scalar array, frequency and entropy, respectively. The $D$ and $B$ terms denote the drift and diffusion coefficients, respectively. The $W$ term represents the Wiener-Lévy processes [48,49]. The system of SDEs models the FDF transport equation (Equation (28)) at the level of one-point one-time statistics and is solved by a Lagrangian Monte Carlo (MC) procedure [43]. Similar to previous FDF simulations [34,43,46,50,51], a hybrid Eulerian/Langrangian numerical solution procedure is being developed in which a Lagrangian setting is used to solve the SDEs. In the Eulerian part, the physical domain is discretized and the filtered continuity, momentum and scalar transport equations are solved via finite difference (FD) or finite volume (FV) methods. The FDF is represented by an ensemble of MC particles. Each of these particles carry information pertaining to the physical field (i.e. all of the FDF variables) and also the position vector. These variables are updated via temporal integration of the modeled SDEs.

\section{Conclusions}

The transport of entropy is introduced in large eddy simulation (LES) to perform more accurate exergy analysis of turbulent combustion systems. The filtered entropy transport equation includes several unclosed terms which represent the effects of entropy generation (exergy destruction). These terms are due to subgrid scale (SGS) viscous dissipation, heat conduction, mass transfer and chemical reaction. A novel methodology based on filtered density function (FDF) is developed for the closure of filtered entropy transport equation. In this methodology, the effects of chemical reaction and its entropy generation contribution appear in closed forms. An exact transport equation is derived for the FDF which is further modeled by a set of stochastic differential equations.

\section{Acknowledgements}

Janbozorgi and Metghalchi were partially supported by Army Research Office under technical monitoring of Ralph Anthenien. 


\section{References}

1. Som, S.K.; Datta, A. Thermodynamic irreversibilities and exergy balance in combustion processes. Prog. Energy Combust. Sci. 2008, 34, 351-376.

2. Rezac, P.; Metghalchi, H. A brief note on the historical evolution and present state of exergy analysis. Int. J. Exergy 2004, 1, 426-437.

3. Nishida, K.; Takagi, T.; Kinoshita, S. Analysis of entropy generation and exergy loss during combustion. Proc. Combust. Inst. 2002, 29, 869-874.

4. Sezer, I.; Altin, I.; Bilgin, A. Exergetic analysis of using oxygenated fuels in spark-ignition (SI) engines. Energ. Fuel. 2009, 23, 1801-1807.

5. Datta, A. Effects of gravity on structure and entropy generation of confined laminar diffusion flames. Int. J. Therm. Sci. 2005, 44, 429-440.

6. Datta, A. Entropy generation in a confined laminar diffusion flame. Combust. Sci. Technol. 2000, 159, 39-56.

7. Som, S.K.; Agrawal, G.K.; Chakraborty, S. Thermodynamics of flame impingement heat transfer. J. Appl. Phys. 2007, 102, 1-9.

8. Stanciu, D.; Marinescu, M.; Dobrovicescu, A. The influence of swirl angle on the irreversibilities in turbulent diffusion flames. Int. J. Thermodyn. 2007, 10, 143-153.

9. Venkatesh, S.; Padiyar, K.S.; Natarajan, R. Second law analysis of methane combustion under different operating conditions. Indian J. Technol. 1987, 25, 506-513.

10. Stanciu, D.; Isvoranu, D.; Marinescu, M.; Gogus, Y. Second law analysis of diffusion flames. Int. J. Appl. Thermody. 2001, 4, 1-18.

11. Briones, A.M.; Mukhopadhyay, A.; Aggarwal, S.K. Analysis of entropy generation in hydrogen-enriched methane-air propagating triple flames. Int. J. Hydrogen Energy 2009, 34, 1074-1083.

12. Li, Z.; Chou, S.; Shu, C.; Yang, W. Entropy generation during micro-combustion. J. Appl. Phys. 2005, 97, 1-8.

13. Raghavan, V.; Gogos, G.; Babu, V.; Sundararajan, T. Entropy generation during the quasi-steady burning of spherical fuel particles. Int. J. Therm. Sci. 2007, 46, 589-604.

14. Polifke, W.; Paschereit, C.O.; Dobbeling, K. Constructive and destructive interference of acoustic and entropy waves in a premixed combustor with a choked exit. Int. J. Acoust. Vibr. 2001, $6,135-146$.

15. Hutchins, T.E.; Metghalchi, M. Energy and exergy analyses of the pulse detonation engine. J. Eng. Gas Turb. Power 2003, 125, 1075-1080.

16. Datta, A.; Som, S. Energy and exergy balance in a gas turbine combustor. Proc. Inst. Mech. Eng. Part A J. Power Energy 1999, 213, 23-32.

17. Rakopoulos, C.D.; Giakoumis, E.G. Second-law analyses applied to internal combustion engines operation. Prog. Energ. Combust. 2006, 32, 2-47.

18. Yapıcı, H.; Kayataş, N.; Albayrak, B.; Baştürk, G. Numerical calculation of local entropy generation in a methane-air burner. Energ. Convers. Manag. 2005, 46, 1885-1919. 
19. Teng, H.; Kinoshita, C.M.; Masutani, S.M.; Zhou, J. Entropy generation in multicomponent reacting flows. J. Energ. Resour.-ASME 1998, 120, 226-232.

20. Lior, N.; Sarmiento-Darkin, W.; Al-Sharqawi, H.S. The exergy fields in transport processes: their calculation and use. Energy 2006, 31, 553-578.

21. Ugarte, S.; Metghalchi, M. Evolution of adiabatic availability and its depletion through irreversible processes. Int. J. of Exergy 2005, 2, 3-13.

22. Chavannavar, P.; Caton, J. Destruction of availability (exergy) due to combustion processes: a parametric study. P. I. Mech. Eng. A-J. Pow. 2006, 220, 655-668.

23. Pope, S.B. Turbulent Flows; Cambridge University Press: Cambridge, UK, 2000.

24. Poinsot, T.; Veynante, D. Theoretical and Numerical Combustion, 2nd ed.; R. T. Edwards, Inc.: Philadelphia, PA, USA, 2005.

25. Janicka, J.; Sadiki, A. Large eddy simulation of turbulent combustion systems. Proc. Combust. Inst. 2005, 30, 537-547.

26. Bilger, R.W. Future progress in turbulent combustion research. Prog. Energy Combust. Sci. 2000, 26, 367-380.

27. Peters, N. Turbulent Combustion; Cambridge University Press: Cambridge, UK, 2000.

28. Menon, S. Subgrid combustion modelling for large-eddy simulations. Int. J. Engine Research 2000, 1, 209-227.

29. Pitsch, H. Large-eddy simulation of turbulent combustion. Annu. Rev. Fluid Mech. 2006, $38,453-482$.

30. Givi, P. Filtered density function for subgrid scale modeling of turbulent combustion. AIAA J. 2006, 44, 16-23.

31. Sheikhi, M.R.H.; Drozda, T.G.; Givi, P.; Jaberi, F.A.; Pope, S.B. Large eddy simulation of a turbulent nonpremixed piloted methane jet flame (Sandia Flame D). Proc. Combust. Inst. 2005, 30, 549-556.

32. Drozda, T.G.; Sheikhi, M.R.H.; Madnia, C.K.; Givi, P. Developments in formulation and application of the filtered density function. Flow Turbul. Combust. 2007, 78, 35-67.

33. Nik, M.B.; Yilmaz, S.L.; Sheikhi, M.R.H.; Givi, P.; Pope, S.B. VSFMDF for large eddy simulation of sandia piloted jet flame. AIAA J. 2010. in press.

34. Sheikhi, M.R.H.; Givi, P.; Pope, S.B. Frequency-velocity-scalar filtered mass density function for large eddy simulation of turbulent flows. Phys. Fluids 2009, 21, 075102 1-14.

35. Givi, P.; Sheikhi, M.R.H.; Drozda, T.G.; Madnia, C.K. Large scale simulation of turbulent combustion. Combust. Plasma Chem. 2008, 6, 1-9.

36. Williams, F.A. Combustion Theory, 2nd ed.; The Benjamin/Cummings Publishing Company: Menlo Park, CA, USA, 1985.

37. Hirschfelder, J.O.; Curtiss, C.F.; Bird, R.B. Molecular Theory of Gases and Liquids; Wiley: New York, NY, USA, 1954.

38. Pope, S.B. The statistical theory of turbulent flames. Phil. Trans. Royal Soc. London 1979, 291, 529-568.

39. Sagaut, P. Large Eddy Simulation for Incompressible Flows; Springer-Verlag: New York, NY, USA, 2001. 
40. Geurts, B.J. Elements of Direct and Large-Eddy Simulation; R. T. Edwards, Inc.: Philadelphia, PA, USA, 2004.

41. Ghosal, S.; Moin, P. The basic equations for the large eddy simulation of turbulent flows in complex geometry. J. Comp. Phys. 1995, 118, 24-37.

42. Vreman, B.; Geurts, B.; Kuerten, H. Realizability conditions for the turbulent stress tensor in large-eddy simulation. J. Fluid Mech. 1994, 278, 351-362.

43. Sheikhi, M.R.H.; Givi, P.; Pope, S.B. Velocity-scalar filtered mass density function for large eddy simulation of turbulent reacting flows. Phys. Fluid. 2007, 19, 095196 1-21.

44. O'Brien, E.E. The probability density function (PDF) approach to reacting turbulent flows. In Turbulent Reacting Flows; Libby, P.A., Williams, F.A., Eds.; Springer-Verlag: Heidelberg, Germany, 1980; Chapter 5, pp. 185-218.

45. Lundgren, T.S. Distribution functions in the statistical theory of turbulence. Phys. Fluid. 1967, 10, 969-975.

46. Sheikhi, M.R.H.; Drozda, T.G.; Givi, P.; Pope, S.B. Velocity-scalar filtered density function for large eddy simulation of turbulent flows. Phys. Fluids 2003, 15, 2321-2337.

47. Karlin, S.; Taylor, H.M. A Second Course in Stochastic Processes; Academic Press: New York, NY, USA, 1981.

48. Wax, N. Selected Papers on Noise and Stochastic Processes; Dover: Mineola, NY, USA, 1954.

49. Gardiner, C.W. Handbook of Stochastic Methods; Springer-Verlag: New York, NY, USA, 1990.

50. Colucci, P.J.; Jaberi, F.A.; Givi, P.; Pope, S.B. Filtered density function for large eddy simulation of turbulent reacting flows. Phys. Fluids 1998, 10, 499-515.

51. Jaberi, F.A.; Colucci, P.J.; James, S.; Givi, P.; Pope, S.B. Filtered mass density function for large eddy simulation of turbulent reacting flows. J. Fluid Mech. 1999, 401, 85-121.

(c) 2010 by the authors; licensee Molecular Diversity Preservation International, Basel, Switzerland. This article is an open-access article distributed under the terms and conditions of the Creative Commons Attribution license http://creativecommons.org/licenses/by/3.0/. 